\title{
25 Research Soure \\ Relapse of undernutrition and food insecurity in a nutritional program in HIV care: mixed-methods study in Tigray region, Ethiopia.
}

Fisaha Haile Tesfay ( $\square$ fisaha.1999@gmail.com )

Mekelle University https://orcid.org/0000-0003-0399-1711

Anna Ziersch

Flinders University Faculty of Medicine Nursing and Health Sciences

Sara Javanparast

Flinders University Faculty of Medicine Nursing and Health Sciences

Lillian Mwanri

Flinders University Faculty of Medicine Nursing and Health Sciences

\section{Research}

Keywords: Food insecurity. Relapse, Mixed methods, Poverty and poor livelihood

Posted Date: January 30th, 2020

DOI: https://doi.org/10.21203/rs.2.22253/v1

License: (1) This work is licensed under a Creative Commons Attribution 4.0 International License. Read Full License 


\section{Abstract}

Introduction

Food insecurity is one of the major contributors to poor attainment of nutritional recovery among people living with HIV who are enrolled in nutritional programs. Food insecurity also significantly contributes to high rates of relapse after nutritional recovery among the beneficiaries nutritional programs. Nevertheless, nutritional programs in HIV care settings implemented in many countries are not designed to address food insecurity. This study examined a nutritional program delivered in HIV care settings in the Tigray region, Ethiopia. It explored the factors that contributed to relapse of undernutrition, and in particular how food insecurity had an impact on effectiveness of the nutritional program, specifically relapse of undernutrition after nutritional recovery.

Methodology

This study employed mixed-methods approach involving quantitative and qualitative methods. In the quantitative part, hospital records were collected of 1757 adults and 236 children people living with HIV who were enrolled in the nutritional program. Logistic and cox-regression analysis were used to examine the frequency and determinants of relapse of undernutrition. In the qualitative study, data was collected through in-depth interviews with 20 adults, 15 caregivers of children living with HIV, and 13 health providers and program managers. Thematic framework analysis was used to analyse the qualitative data.

Results

Among those who graduated from the nutritional program, $18 \%$ of adults and $7 \%$ of children relapsed to undernutrition. The mean time to relapse for adults was 68.5 months (95\% $\mathrm{Cl}, 67.0-69.9)$. It was not possible to calculate the average time to relapse in children because of the small sample size. Lower educational status (primary and secondary), no membership of a community HIV support group, ambulatory and bedridden functional status, longer periods on ART (more than 24 months), presence of an opportunistic infection and poor baseline nutritional status were associated with relapse of undernutrition. Furthermore, those from rural areas, who did not attend formal education, were employed, and had bedridden functional status, anemia and worst nutritional status were likely to have more frequent episodes of relapse than their counterparts. Findings of the qualitative study also highlighted that poverty, poor livelihood, and food insecurity were the fundamental challenges to the effectiveness of nutritional programs in HIV care. Household food insecurity contributed to the selling and sharing of the nutritional supports and negatively impacted the program effectiveness, delaying nutritional recovery and contributing to relapse of undernutrition.

Conclusion and recommendation

Unless nutritional programs take into consideration the underlying determinants of food insecurity in the design, implementation, and funding of nutritional programs in HIV care, the success of the nutritional programs like those implemented in Ethiopia will be undermined and the vicious cycle of undernutrition amongst people living with HIV will continue.

\section{Background}

Household food insecurity is generally defined as "the ability of the household to acquire the food needed by its members" [1]. Food insecurity is a broad concept with varying indicators across context and usually depends on the target population and scale of measurements - for example, country, household and individual levels [2]. Broadly, food 
security encompasses the quantity and quality of food accessed by a country, household or an individual [1, 2]. The most common measure of household food insecurity among people living with HIV/AIDS in sub-Saharan Africa is the Household Food Insecurity Access scale (HFIAS) [2]. HFIAS is a simple tool (questionnaire) designed to measure (assess, evaluate and monitor) the level of access of households to basic food [3] but it can be customised to specific countries or contexts.

Food insecurity and human immunodeficiency virus/acquired immune deficiency syndrome (HIV/AIDS) are highly linked. Evidence suggests a high prevalence of food insecurity among people living with HIV [4-6]. For example, Nagata and colleagues found that $79 \%$ and $21 \%$ of HIV patients in a study conducted in Kenya were severely and moderately food insecure respectively [6]. Similarly, a study conducted in Ethiopia reported food insecurity among $63 \%$ of people living with HIV enrolled in HIV care [7].

Food insecurity has a range of impacts on people living with HIV such as undernutrition, non-adherence to ART and poor HIV treatment outcomes [5, 8, 9]. Research has identified food insecurity as a key barrier to ART adherence among people living with HIV $[5,10]$. Food insecurity can also increase susceptibility to HIV acquisition due to affected individuals' engagement in risky sexual practices to overcome issues of food insecurity [11]. HIV infection, on the other hand, increases susceptibility to food insecurity because of loss of economic productivity and increased dependency of those living with HIV $[12,13]$. In addition, in areas severely affected by the epidemic, HIV/AIDS-related mortality and morbidity results in household labour shortage, a decline in household assets, loss of essential skills and an increasing cost for the care of sick adults and orphaned children [14].

The overall aim of nutritional programs in HIV care settings is to improve nutritional status, HIV treatment outcomes, and ART adherence for people living with HIV. The types of nutritional programs and mechanisms for their implementation vary widely across countries. Many nutritional programs in sub-Saharan Africa provide ready to use therapeutic foods (RUTF) or Ready to Use supplementary foods (RUSF) for a short period of time to tackle undernutrition amongst people living with $\operatorname{HIV}[15,16]$. Alongside nutritional support, nutritional assessment and counseling are the essential components of nutritional programs in HIV care in sub-Saharan Africa [17].

Clinic-based short term food assistance programs have been proven to be beneficial for food-insecure HIV patients to improve ART adherence and temporary weight gain but do not sustainably overcome the long term nutritional needs of people living with HIV [18-21]. The absence of long term positive impact of food assistance on nutritional status among people living with HIV may be related to a narrow focus of the programs.

Various demographic and socioeconomic factors contribute to food insecurity among people living with HIV. Sociodemographic factors such as household family size, marital status (not married) and older age were associated with food insecurity among people living with HIV in Kenya [6]. An Ethiopian study reported that elementary or lower educational status, below-average monthly family income, and lower food diversity were associated with food insecurity among people living with HIV who were on ART $[7,22]$. Other studies from other parts of sub-Saharan Africa have also shown that lower educational status, absence of nutritional support and low socioeconomic status were associated with poorer food insecurity $[23,24]$.

Research suggests that food insecurity is one of the major contributors to low attainment of nutritional recovery among people living with HIV enrolled in a nutritional care $[25,26]$. Drop out (default) from the nutritional program in HIV care settings have also been found to be related to household food insecurity in sub-Saharan Africa $[25,26]$. However, despite the high magnitude of food insecurity amongst people living with HIV and the role of demographic and socioeconomic issues to undernutrition, nutritional programs in HIV care settings are not designed to respond to such issues. 
Nutritional programs in HIV care neither consider nor assess individual or household food insecurity when enrolling people living with HIV into nutritional programs. For this reason, maintaining nutritional status after nutritional recovery is a major challenge for both adults and children enrolled in the programs [25-28]. A study from Ethiopia showed that nutritional programs in HIV care settings were characterised by high (20\%) relapse of undernutrition after nutritional recovery [25]. A small number of studies have highlighted that sharing and selling of the nutritional support are common practices among food insecure households $[25,29]$. However, none of these studies have examined the impact of food insecurity on the effectiveness of the nutritional program, particularly when it comes to relapse of undernutrition. In addition, in a study that examined the use, perception, and acceptability of a nutritional support in HIV care, food insecurity was common among those enrolled in the nutritional program, though the impact on effectiveness of the nutritional programs was not explored [30]. Overall, there is a lack of evidence on how food insecurity may influence the effectiveness of nutritional programs in HIV care in Ethiopia-particularly in relation to relapse of undernutrition after nutritional recovery. Hence, this study aims to address this gap by examining the extent

and predictors of relapse of undernutrition for patients enrolled in the nutritional program in HIV care in Ethiopia, with a particular focus on how food insecurity contributed to the problems of effectiveness of the nutritional program in terms of relapse of undernutrition.

\section{Methods And Settings}

\section{Description of the nutritional program and study settings}

This study was conducted in three purposively selected general hospitals (Mekelle, Lemlem Karl, and Shul) in the Tigray region, Ethiopia. In the Tigray region, there were 37 hospitals including 20 primary district hospitals, 15 general hospitals and 2 regional referral hospitals at the date of data collection (August 2016) [31]. The three hospitals were selected based on the distance from where health service was managed, coordinated and supervised in Tigray region. One hospital (Mekelle hospital) was the closest and located in Mekelle city where the health system in the region was managed and supervised while the remaining two (hospitals Shul and Lemlem Karl)were far from Mekelle city with approximately comparable distance from the centre. The selection of these hospitals was also made to minimize double-counting due to inter-hospital transfer of the study participants.

In Ethiopia, all HIV patients enrolled in chronic HIV care are regularly screened for undernutrition during their follow up visits for HIV care services. After enrolment in the nutritional program, sociodemographic, clinical, anthropometric, and nutritional outcome data is collected for monitoring and provision of RUTF or RUSF nutritional support. The enrolment and exit criteria and ration sizes are different for children and adult HIV patients enrolled in the nutritional program. While RUTF is given to individuals with severe acute undernutrition, RUSF is provided to moderate and mild undernutrition. Depending on the severity of undernutrition, adult and child patients are provided with two sachets of RUSF daily for a maximum of three months for moderate acute malnutrition (MAM) or body mass index (BMI) of $\leq$ 17.99. For individuals with severe acute undernutrition, four sachets of RUTF daily for a maximum of six months for severe acute undernutrition (SAM) or $\mathrm{BMI} \leq 16$, together with counselling is provided [25, 32].

An HIV patient (adult or a child) enrolled in the nutritional program is recorded as having an outcome of 'recovery', 'nonresponse', 'default' or 'relapse' after nutritional support for the program durations, summarised below (Table 1): 
Nutritional program outcomes in the nutritional program in Ethiopia.

\section{Outcome Definition}

Adults Children

\begin{tabular}{|lll|}
\hline Recovery & $\begin{array}{l}\text { Reaching BMI of } 18.5 \mathrm{~kg} / \mathrm{m}^{2} \\
\text { for two consecutive visits }\end{array}$ & $\begin{array}{l}\text { For children } 6 \text { months to } 14.9 \text { years, weight for length percent }(\mathrm{W} / \mathrm{L} \\
\text { \%) or weight for height percent }(\mathrm{W} / \mathrm{H} \geq 85 \%) \text { on more than one } \\
\text { occasion and absence of oedema for } 10 \text { days }\end{array}$ \\
\hline $\begin{array}{l}\text { Non- } \\
\text { response }\end{array}$ & $\begin{array}{l}\text { Participant did not reach a } \\
\text { BMl of } 18.5 \mathrm{~kg} / \mathrm{m} 2 \text { for two } \\
\text { consecutive visits }\end{array}$ & $\begin{array}{l}\text { All children who do not fulfill the above recovery / discharge criteria } \\
\text { for the duration }\end{array}$ \\
\hline Default & $\begin{array}{l}\text { Did not reach a BMl of } \\
18.5 \mathrm{~kg} / \mathrm{m} 2 \text { and dropped out } \\
\text { of the program }\end{array}$ & $\begin{array}{l}\text { Child dropped out of the program before meeting the criteria for } \\
\text { recovery }\end{array}$ \\
\hline Relapse & $\begin{array}{l}\text { Participants return either with } \\
\text { SAM or MAM after declared } \\
\text { nutritionally recovered }\end{array}$ & $\begin{array}{l}\text { Child returned with either SAM or MAM after declared recovery and } \\
\text { discharged from the nutritional program }\end{array}$ \\
\hline
\end{tabular}

\section{Study design}

This study employed a concurrent mixed-methods approach where the sequence of data collection for both the quantitative and qualitative study was irrelevant but intended to complement each other [33-35], with mixing of the two studies in the interpretation stage.

\section{Sample size and recruitment}

Quantitative and qualitative data were collected between March and July 2016. Quantitative data included records of 1756 adults and 236 children living with HIV (from November 2010 to February 2016). Data were retrieved from a paper-based database in the three selected hospitals. Records with no baseline demographic, socioeconomic, clinical, immunological, nutritional and anthropometric characteristics were excluded - a total of 24 adult and 9 child records.

The qualitative study involved in-depth interviews with 48 purposively selected individuals - 20 adults living with HIV, 15 caregivers of children living with HIV, 11 health providers, and 2 program managers. Adults and caregivers were recruited for the in-depth interviews at the health facility during their regular follow up for HIV services including the nutritional program. Health providers provided information to them about the project inviting them to contact the researcher to participate in the research if they were interested. They were assured that their participation or otherwise in the research would be confidential and have no impact on their access to services. Health providers and program managers were identified as working in the program for more than 1 year and invited to participate in the research by email, where they could contact the researcher if they would like to be involved. Participant recruitment continued until data saturation was achieved. Interviews were conducted at a venue convenient to participants, largely the HIV service while the program managers were interviewed at their workplace (Tigray regional Health Bureau head office).

\section{Data collection procedure}

Nine data collectors (three in each hospital) familiar with the hospital data management system were recruited and trained to collect quantitative data. Two different checklists for adults and children were designed covering 
demographic, socioeconomic, clinical, immunological, nutritional and anthropometric variables. Data were retrieved from multiple sources enabling cross-matching and included: a) nutritional program follow up form; b) HIV care follow up form, and c) ART pharmacy database. Unique ART and pre-ART number were used to match or relate data from the various sources

For the qualitative data, interview guides were developed covering issues around participants' knowledge of the nutritional program and factors enabling or constraining program utilisation. The interview guide was field-tested and further refined based on emerging concepts and ideas. In-depth interviews were conducted by the first author (FT) in the local language Tigrigna and audio-recorded. Comprehensive field notes were taken during the fieldwork.

\section{Data management and analysis}

Quantitative data analysis involved description of the demographic, socioeconomic, clinical, immunological, nutritional and anthropometric characterises of adult and child records. In addition, description of the outcome variables such as relapses of undernutrition and frequency of relapse of undernutrition were done. For relapses of undernutrition after nutritional recovery, Kaplan Meier curve was used to estimate the average time to relapses.

To identify the baseline determinants of relapse of undernutrition, bivariate Cox regression analysis was used to estimate the crude hazard ratio. Independent variables which were statistically significant at $p<0.3$ were taken to multivariate cox regression to estimate the adjusted hazard ratio, with 95\% $\mathrm{Cl}$. In multivariate Cox regression, statistical significance was declared at $p<0.05$ and the hazard ratio was used to interpret the results.

The second outcome variable was the frequency of relapse of undernutrition. To identify the determinants of frequency of relapse of undernutrition, bivariate logistic regression was conducted to estimate the crude odds ratio and statistical significance was considered at $p$-value $<0.3$. Assumptions of logistic regression such as multicollinearity and homogeneity of variance were checked and no collinearity was identified. Factors that were found to be statistically significant at $p<0.3$ were taken to multivariate logistic regression model to determine the independent predictors of the frequency of relapse. Adjusted odds ratio with $95 \% \mathrm{Cl}$ were used to determine the magnitude and direction of relationship and statistical significance was considered at $p<0.05$.

Qualitative interviews were translated and transcribed into English by the first author (FT). Framework thematic analysis [36] was used to analyse the qualitative data. A coding framework was developed and discussed in the team meetings until a consensus was reached among the authors. Three interviews were double coded by the authors and differences were discussed until consensus was reached. Data was analysed using QSR NVivo and themes and categories emerged after thorough reading and understanding of the interview data, field notes, and memos. Illustrative quotes are used to describe themes and categories.

\section{Results}

\section{Demographic and socioeconomic characteristics of adults and children living with HIV}

The most common age group for adults was the 26-35 age group (42.6\%), 63\% were female and two-thirds lived in urban areas (Table 2). More than $40 \%$ were married, and over three quarters $(76.6 \%)$ had children, with the majority of people living in a household of less than 5 people. Nearly $62 \%$ of adults attended at least primary and secondary education and a large proportion of adults and parents of children living with HIV were unemployed. The vast majority 
of people reported their religion as Orthodox or other Christian religion. Over three quarters had disclosed their positive HIV status to at least someone, and $81 \%$ of adults were not members of a community HIV support group.

The most common age group for children was $5-10$ years $(43.2 \%)$ and $47.5 \%$ were female. The vast majority of children (82.6\%) of children lived with their parents (of whom more than half were living together), and three quarters lived in urban areas. There were similar proportions of the children who were the first, second, third and fourth-child in their family. Most children attended school. 
Table 2

Demographic and socioeconomic of adults and children enrolled in the nutritional program.

\begin{tabular}{|c|c|c|c|c|c|c|c|}
\hline \multicolumn{4}{|c|}{ Adults ( $N=1757)$} & \multicolumn{4}{|c|}{ Children $(N=236)$} \\
\hline Variables & Categories & Number & Percent & Variables & Categories & Percent & Number \\
\hline \multirow[t]{4}{*}{ Age } & $<25$ & 216 & 12.3 & \multirow[t]{3}{*}{ Age } & $<5$ years & 62 & 26.3 \\
\hline & $26-35$ & 749 & 42.6 & & $5-10$ years & 102 & 43.2 \\
\hline & $36-45$ & 531 & 30.2 & & $>10$ years & 72 & 30.5 \\
\hline & $>46$ & 261 & 14.9 & \multirow[t]{2}{*}{ Sex } & Male & 124 & 52.5 \\
\hline \multirow[t]{2}{*}{ Sex } & Male & 649 & 36.9 & & Female & 112 & 47.5 \\
\hline & Female & 1108 & 63.1 & \multirow[t]{2}{*}{ Residence } & Urban & 179 & 75.8 \\
\hline \multirow[t]{2}{*}{ Residence } & Urban & 1171 & 66.6 & & Rural & 57 & 24.2 \\
\hline & Rural & 586 & 33.4 & \multirow{5}{*}{$\begin{array}{l}\text { Child lives } \\
\text { with }\end{array}$} & Parents & 195 & 82.6 \\
\hline \multirow[t]{5}{*}{ Marital status } & Never married & 265 & 15.1 & & Gurdian & 15 & 6.4 \\
\hline & $\begin{array}{l}\text { Married (In. } \\
\text { defacto) }\end{array}$ & 722 & 41.1 & & $\begin{array}{l}\text { Grand } \\
\text { parents }\end{array}$ & 2 & 0.8 \\
\hline & Separated & 164 & 9.3 & & $\begin{array}{l}\text { In } \\
\text { orphanage }\end{array}$ & 14 & 5.9 \\
\hline & Divorced & 381 & 21.7 & & Sibilings & 10 & 4.2 \\
\hline & Widow/Widower & 225 & 12.8 & \multirow{5}{*}{$\begin{array}{l}\text { Marital } \\
\text { status of } \\
\text { parents, } n= \\
195\end{array}$} & $\begin{array}{l}\text { Mother and } \\
\text { father live } \\
\text { together }\end{array}$ & 100 & 51.3 \\
\hline \multirow{4}{*}{$\begin{array}{l}\text { Education } \\
\text { status }\end{array}$} & No education & 535 & 30.4 & & Divorced & 16 & 8.2 \\
\hline & Primary & 637 & 36.3 & & Widowed & 5 & 2.7 \\
\hline & Secondary & 459 & 26.1 & & $\begin{array}{l}\text { Single } \\
\text { parent } \\
\text { father }\end{array}$ & 18 & 9.2 \\
\hline & Tertiary & 126 & 7.2 & & $\begin{array}{l}\text { Single } \\
\text { parent } \\
\text { mother }\end{array}$ & 54 & 27.7 \\
\hline \multirow[t]{2}{*}{ Religion } & $\begin{array}{l}\text { Orthodox and } \\
\text { other Christian }\end{array}$ & 1659 & 94.4 & \multirow[t]{2}{*}{$\begin{array}{l}\text { Mother alive, } \\
n=208\end{array}$} & Yes & 175 & 84.1 \\
\hline & Muslim & 98 & 5.6 & & No & 33 & 15.9 \\
\hline \multirow[t]{3}{*}{ Employment } & Working & 871 & 50.8 & \multirow{2}{*}{$\begin{array}{l}\text { Emploment } \\
\text { status of } \\
\text { mother if } \\
\text { alive. } n=147\end{array}$} & Employed & 55 & 37.4 \\
\hline & $\begin{array}{l}\text { Employed but } \\
\text { not working due } \\
\text { to ill health }\end{array}$ & 111 & 6.5 & & Unemployed & 92 & 62.6 \\
\hline & Unemployed & 732 & 42.7 & \multirow{2}{*}{$\begin{array}{l}\text { Father alive, } \\
n=202\end{array}$} & Yes & 146 & 72.3 \\
\hline \multirow{2}{*}{$\begin{array}{l}\text { Household } \\
\text { family size }\end{array}$} & $\leq 5$ & 1543 & 87.8 & & No & 56 & 27.7 \\
\hline & $>5$ & 207 & 11.8 & Employment & Employed & 90 & 71.4 \\
\hline
\end{tabular}




\begin{tabular}{|c|c|c|c|c|c|c|c|}
\hline \multicolumn{4}{|l|}{ Adults $(\mathrm{N}=1757)$} & \multicolumn{4}{|c|}{ Children $(\mathrm{N}=236)$} \\
\hline \multirow[t]{2}{*}{ Have children } & Yes & 1346 & 76.6 & $\begin{array}{l}\text { status of } \\
\text { father if } \\
\text { alive. } n=126\end{array}$ & Unemployed & 36 & 28.6 \\
\hline & No & 411 & 23.4 & \multirow{4}{*}{$\begin{array}{l}\text { Child's birth } \\
\text { order }\end{array}$} & First & 32 & 13.6 \\
\hline \multirow{2}{*}{$\begin{array}{l}\text { Membership of } \\
\text { HIV related } \\
\text { community } \\
\text { support group }\end{array}$} & Yes & 333 & 19.0 & & Second & 42 & 17.8 \\
\hline & No & 1424 & 81.0 & & Third & 32 & 13.6 \\
\hline \multirow[t]{2}{*}{$\begin{array}{l}\text { Disclosure to at } \\
\text { least someone }\end{array}$} & Yes & 1366 & 77.7 & & $\begin{array}{l}\text { Fourth and } \\
\text { above }\end{array}$ & 28 & 11.9 \\
\hline & No & 391 & 22.3 & \multirow{2}{*}{$\begin{array}{l}\text { Child atted } \\
\text { school }\end{array}$} & Yes & 170 & 72.0 \\
\hline \multirow{6}{*}{$\begin{array}{l}\text { Name of } \\
\text { hospital }\end{array}$} & Mekelle & 1045 & 59.5 & & No & 66 & 28.0 \\
\hline & \multirow[t]{2}{*}{ Lemlem Karl } & \multirow[t]{2}{*}{378} & \multirow[t]{2}{*}{21.5} & \multirow{2}{*}{$\begin{array}{l}\text { Reason for } \\
\text { not atteding } \\
\text { school, } n=66\end{array}$} & Too young & 60 & 90.1 \\
\hline & & & & & $\begin{array}{l}\text { Lack of } \\
\text { fund }\end{array}$ & 6 & 9.1 \\
\hline & \multirow[t]{3}{*}{ Shul Hospital } & \multirow[t]{3}{*}{334} & \multirow[t]{3}{*}{19.0} & \multirow{3}{*}{$\begin{array}{l}\text { Name of } \\
\text { hospital }\end{array}$} & Mekelle & 189 & 80.1 \\
\hline & & & & & $\begin{array}{l}\text { Lemlem } \\
\text { Karl }\end{array}$ & 24 & 10.2 \\
\hline & & & & & Shul & 23 & 9.7 \\
\hline
\end{tabular}

\section{Clinical and immunological characteristics of adults and children living with HIV}

The functional status of the majority of adult participants was 'working' (83.8\%), while $50.5 \%$ of adults and $56 \%$ of children were in WHO clinical stage I. Furthermore, $94 \%$ of adults and $88 \%$ of children were on ART at baseline or at the time of enrolment in the nutritional program. In addition, $45.7 \%$ of adults were anaemic at enrolment. $64 \%$ of adults and $72 \%$ of children had been on ART for 24 months at enrolment to the nutritional program. A quarter (25\%) of adults and $22 \%$ of children had opportunistic infections, with TB being the major opportunistic infection in both groups (Table 3). 
Table 3

Clinical and immunological characteristics of adults and children enrolled in the nutritional program.

\begin{tabular}{|c|c|c|c|c|c|c|c|}
\hline \multicolumn{4}{|l|}{ Adults ( $\mathrm{N}=236)$} & \multicolumn{4}{|l|}{ Children $(\mathrm{N}=236)$} \\
\hline \multicolumn{2}{|l|}{ Variables } & \multirow{2}{*}{$\begin{array}{l}\text { Number } \\
1473\end{array}$} & \multirow{2}{*}{$\begin{array}{l}\text { Percent } \\
83.8\end{array}$} & \multicolumn{4}{|l|}{ Variables } \\
\hline \multirow[t]{3}{*}{ Functional status } & Working & & & \multirow[t]{4}{*}{ WHO clinical stage } & Stage I & 132 & 55.9 \\
\hline & Ambulatory & 207 & 11.8 & & Stage II & 42 & 17.8 \\
\hline & Bedridden* & 77 & 4.4 & & Stage III & 50 & 21.2 \\
\hline \multirow[t]{4}{*}{ WHO clinical stage } & Stage I & 888 & 50.5 & & Stage IV & 12 & 5.1 \\
\hline & Stage II & 225 & 12.8 & \multirow{2}{*}{$\begin{array}{l}\text { ART status at } \\
\text { enrolment }\end{array}$} & On pre-ART & 29 & 12.3 \\
\hline & Stage III & 518 & 29.5 & & On ART & 207 & 87.7 \\
\hline & Stage IV* & 126 & 7.2 & \multirow[t]{3}{*}{ Duration on ART } & $<12$ months & 27 & 13.0 \\
\hline \multirow[t]{4}{*}{ Baseline CD4 count } & $<200$ & 675 & 39.0 & & $\begin{array}{l}13-24 \\
\text { months }\end{array}$ & 31 & 15.0 \\
\hline & $200-349$ & 488 & 28.2 & & $>24$ months & 149 & 72.0 \\
\hline & $350-500$ & 294 & 17.0 & \multirow{2}{*}{$\begin{array}{l}\text { Contrimoxzole } \\
\text { propylaxis }\end{array}$} & Yes & 209 & 88.6 \\
\hline & $>500$ & 274 & 15.8 & & No & 27 & 11.1 \\
\hline \multirow[t]{2}{*}{ Presence of anaemia } & Anaemic & 687 & 45.7 & \multirow{2}{*}{$\begin{array}{l}\text { Presence of } \\
\text { opportunistic } \\
\text { infection }\end{array}$} & Yes & 52 & 22.0 \\
\hline & $\begin{array}{l}\text { Not } \\
\text { Anaemic }\end{array}$ & 815 & 54.3 & & No & 184 & 78.0 \\
\hline \multirow[t]{2}{*}{ ART status } & Pre-ART & 100 & 5.7 & \multirow{3}{*}{$\begin{array}{l}\text { Type of } \\
\text { opportunistic } \\
\text { infection }\end{array}$} & TB & 14 & 26.9 \\
\hline & ART & 1657 & 94.3 & & TB and other & 10 & 19.2 \\
\hline \multirow[t]{4}{*}{ Duration on ART } & $<6$ months & 324 & 19.6 & & Other & 28 & 53.8 \\
\hline & $\begin{array}{l}6-12 \\
\text { months }\end{array}$ & 99 & 6.0 & \multirow[t]{3}{*}{$\begin{array}{l}\text { Eligibility criteria to } \\
\text { ART }\end{array}$} & $\begin{array}{l}\text { WHO clinical } \\
\text { stage only }\end{array}$ & 28 & 11.9 \\
\hline & $\begin{array}{l}12-24 \\
\text { months }\end{array}$ & 175 & 10.6 & & $\begin{array}{l}\text { CD4 or TLC } \\
\text { only }\end{array}$ & 15 & 58.5 \\
\hline & $\begin{array}{l}>24 \\
\text { months }\end{array}$ & 1059 & 63.9 & & CD4\% only & 47 & 19.9 \\
\hline \multirow{2}{*}{$\begin{array}{l}\text { Presence of } \\
\text { opportunistic infection }\end{array}$} & Yes & 436 & 24.8 & & & & \\
\hline & No & 1321 & 75.2 & & & & \\
\hline \multirow{3}{*}{$\begin{array}{l}\text { Type of opportunistic } \\
\text { infection }\end{array}$} & TB & 152 & 37.3 & & & & \\
\hline & $\begin{array}{l}\text { TB and } \\
\text { others }\end{array}$ & 47 & 11.5 & & & & \\
\hline & Others & 208 & 51.1 & & & & \\
\hline
\end{tabular}




\section{Nutritional and anthropometric characteristics of adult and child participants}

Before enrolment into the nutritional program, $62.5 \%$ of adults and $80 \%$ of children participants had moderate undernutrition, while $23 \%$ of adults and $8 \%$ of children had severe acute undernutrition. Almost $78 \%$ of adults received less than three sachets per day during their engagement with the program. Having good appetite was one of the criteria for enrolment into the nutritional program and almost all of the adults reported having good appetite. Those with poor appetite were treated for the cause before enrolment into the nutritional program. Usually, the causes of poor appetite were opportunistic infections (Table 4).

After enrolment in the nutritional program, $55 \%$ of adults and $71 \%$ of children recovered or achieved the graduation criteria of a target BMI. Non-response (not meeting target BMI) was identified in $21 \%$ of adults and $14 \%$ of children. Amongst the study cohort, $18 \%$ of adults and $14 \%$ of children failed to complete the program. Among those who achieved nutritional recovery, $18 \%$ of adults and $7 \%$ of children later relapsed (Table 4 ). 
Table 4

Nutritional characteristics of adults and children enrolled in the nutritional program

\begin{tabular}{|c|c|c|c|c|c|c|c|}
\hline \multicolumn{4}{|c|}{ Adults ( $N=1757)$} & \multicolumn{4}{|c|}{ Children $(\mathrm{N}=236)$} \\
\hline Variables & & Number & Percent & Variables & & Number & Percent \\
\hline \multirow{3}{*}{$\begin{array}{l}\text { Nutritional } \\
\text { status at } \\
\text { enrolment }\end{array}$} & $\begin{array}{l}\text { Mild acute } \\
\text { undernutrition }\end{array}$ & 253 & 14.4 & \multirow{3}{*}{$\begin{array}{l}\text { Nutritional } \\
\text { status at } \\
\text { enrolment }\end{array}$} & $\begin{array}{l}\text { Mild acute } \\
\text { undernutrition }\end{array}$ & 29 & 12.3 \\
\hline & $\begin{array}{l}\text { Moderate acute } \\
\text { undernutrition }\end{array}$ & 1098 & 62.5 & & $\begin{array}{l}\text { Moderate } \\
\text { acute } \\
\text { undernutrition }\end{array}$ & 188 & 79.7 \\
\hline & $\begin{array}{l}\text { Severe acute } \\
\text { undernutrition }\end{array}$ & 406 & 23.1 & & $\begin{array}{l}\text { Severe acute } \\
\text { undernutrition }\end{array}$ & 19 & 8.1 \\
\hline \multirow{2}{*}{$\begin{array}{l}\text { Number of } \\
\text { sachets/ } \\
\text { day }\end{array}$} & $\leq 3$ & 1362 & 77.6 & \multirow{3}{*}{$\begin{array}{l}\text { Duration on } \\
\text { nutritional } \\
\text { program }\end{array}$} & $<3$ months & 28 & 11.9 \\
\hline & $\geq 4$ & 394 & 22.4 & & 3 months & 193 & 81.8 \\
\hline \multirow{2}{*}{$\begin{array}{l}\text { Appetite } \\
\text { test done }\end{array}$} & Yes & 1731 & 98.5 & & $>3$ months & 15 & 6.4 \\
\hline & No & 26 & 1.5 & \multirow{5}{*}{$\begin{array}{l}\text { Nutritional } \\
\text { outcome }\end{array}$} & Graduated & 167 & 70.8 \\
\hline \multirow[t]{2}{*}{$\begin{array}{l}\text { Appetite } \\
\text { test results }\end{array}$} & Good* & 1150 & 65.5 & & $\begin{array}{l}\text { Non- } \\
\text { respondent }\end{array}$ & 33 & 14.0 \\
\hline & Poor & 580 & 33.0 & & Defaulted & 33 & 13.9 \\
\hline \multirow{2}{*}{$\begin{array}{l}\text { Subsequent } \\
\text { weight gain }\end{array}$} & Good* & 1650 & 93.9 & & Died & 2 & 0.8 \\
\hline & Poor & 70 & 4.0 & & $\begin{array}{l}\text { Transferred } \\
\text { out }\end{array}$ & 1 & 0.4 \\
\hline \multirow{5}{*}{$\begin{array}{l}\text { Nutritional } \\
\text { outcome }\end{array}$} & Graduated/recovered & 971 & 55.3 & \multirow{2}{*}{$\begin{array}{l}\text { Relapse of } \\
\text { malnutrition }\end{array}$} & Yes & 12 & 7.2 \\
\hline & Non-respondent & 379 & 21.0 & & No & 154 & 92.8 \\
\hline & $\begin{array}{l}\text { In } \\
\text { completed(Defaulted) }\end{array}$ & 329 & 18.7 & & & & \\
\hline & Death & 35 & 2.0 & & & & \\
\hline & Transferred out & 43 & 2.4 & & & & \\
\hline \multirow{2}{*}{$\begin{array}{l}\text { Relapse } \\
\text { after } \\
\text { nutritional } \\
\text { recovery (n } \\
=968)\end{array}$} & Yes & 170 & 17.6 & & & & \\
\hline & No & 801 & 82.4 & & & & \\
\hline
\end{tabular}

\section{Determinants of relapses of undernutrition}

The total time to relapse after nutritional recovery in the nutritional program was 68.5 months (95\% $\mathrm{Cl} 67.0-69.9)$. We conducted bivariate and multivariate Cox regression to identify the independent predictors of relapses of undernutrition after nutritional recovery.

In multivariate Cox regression, statistical significance was declared at $\mathrm{P}<0.05$. Accordingly, educational status, membership of an HIV community support group, duration on ART, ambulatory and bedridden functional status, presence of opportunistic infections, moderate and severe acute undernutrition were significantly associated with 
relapse of undernutrition after nutritional recovery. Adults who attended primary and secondary education were 2.8 and 3.7 times more likely to relapse than those who attended tertiary and above, but there was no difference for those who had no education. Non-membership of HIV community support group was associated with a 1.7 times higher chance of relapse than those who were members of community HIV support group. In addition, those who had been on ART for longer periods were more likely to relapse than those who had been less than 24 months on ART. Adults who had opportunistic infections at baseline were 1.7 times the risk of relapse of undernutrition after nutritional recovery (Table 5). 
Table 5

Determinants of relapse of undernutrition after nutritional recovery

\begin{tabular}{|c|c|c|c|c|c|c|}
\hline \multirow[t]{3}{*}{ Variables } & & \multicolumn{2}{|c|}{ Relapse of malnutrition } & \multirow{3}{*}{$\begin{array}{l}\text { Crude Hazard } \\
\text { ratio }(95 \% \mathrm{Cl})\end{array}$} & \multirow{3}{*}{$\begin{array}{l}\text { Adjusted } \\
\text { Hazard ratio } \\
(95 \% \mathrm{Cl})\end{array}$} & \multirow{3}{*}{$\begin{array}{l}\mathrm{P}- \\
\text { value }\end{array}$} \\
\hline & & \multirow{2}{*}{$\begin{array}{l}\text { No } \\
\text { No (\%) }\end{array}$} & \multirow{2}{*}{$\begin{array}{l}\text { Yes } \\
\text { No (\%) }\end{array}$} & & & \\
\hline & & & & & & \\
\hline \multirow[t]{2}{*}{ Sex } & Male & 289(83.5) & $57(16.5)$ & $\begin{array}{l}0.92(0.67- \\
1.27)\end{array}$ & $\begin{array}{l}0.91(0.64- \\
1.29)\end{array}$ & 0.591 \\
\hline & Female & $509(81.8)$ & 113(18.2) & 1.0 & 1.0 & \\
\hline \multirow[t]{2}{*}{ Residence } & Urban & $533(81.5)$ & 121(18.5) & $\begin{array}{l}1.16(0.83- \\
1.62)\end{array}$ & $\begin{array}{l}0.94(0.65- \\
1.34)\end{array}$ & 0.740 \\
\hline & Rural & $265(84.4)$ & 49(15.6) & 1.0 & 1.0 & \\
\hline \multirow[t]{4}{*}{ Marital status } & Single & $131(86.8)$ & 21(13.2) & 1.0 & 1.0 & \\
\hline & Married & $342(83.2)$ & $69(16.8)$ & $\begin{array}{l}1.33(0.81- \\
2.19)\end{array}$ & $\begin{array}{l}1.27(0.76- \\
2.13)\end{array}$ & 0.363 \\
\hline & Divorced & $228(80.9)$ & $54(19.1)$ & $\begin{array}{l}1.49(0.89- \\
2.49)\end{array}$ & $\begin{array}{l}1.50(0.88- \\
2.56)\end{array}$ & 0.132 \\
\hline & Widowed & $97(78.2)$ & $27(21.8)$ & $\begin{array}{l}1.57(0.88- \\
2.82)\end{array}$ & $1.36(0.74-$ & 0.331 \\
\hline \multirow[t]{4}{*}{ Educational status } & No education & 231(84.9) & $41(15.1)$ & $\begin{array}{l}1.055(0.75- \\
1.49)\end{array}$ & $\begin{array}{l}2.84(0.87- \\
9.26)\end{array}$ & 0.080 \\
\hline & Primary & $276(79.8)$ & $70(20.2)$ & $\begin{array}{l}1.48(1.07- \\
2.03)\end{array}$ & $\begin{array}{l}3.68(1.15- \\
11.77)\end{array}$ & 0.033 \\
\hline & Secondary & $276(79.8)$ & $70(20.2)$ & $1.39(1.0-1.93)$ & $\begin{array}{l}3.25(1.01- \\
10.48) *\end{array}$ & 0.049 \\
\hline & Tertiary & 291(83.1) & $59(16.9)$ & 1.0 & 1.0 & \\
\hline \multirow[t]{2}{*}{ Employment } & Working & $425(84.7)$ & $77(15.30$ & 1.0 & 1.00 & \\
\hline & Not working & $353(79.3)$ & $92(20.7)$ & $\begin{array}{l}1.36(1.00- \\
1.84)\end{array}$ & $\begin{array}{l}1.32(0.97- \\
1.81)\end{array}$ & 0.08 \\
\hline \multirow{2}{*}{$\begin{array}{l}\text { Membership of } \\
\text { community support }\end{array}$} & Yes & 127(73.0) & $47(27.0)$ & 1.0 & 1.0 & \\
\hline & No & $671(84.50)$ & $123(15.5)$ & $\begin{array}{l}1.87(1.33- \\
2.63)\end{array}$ & $\begin{array}{l}1.78(1.25- \\
2.54) *\end{array}$ & 0.001 \\
\hline \multirow[t]{2}{*}{ Functional status } & Working & 694(81.9) & 153(18.1) & 1.0 & 1.0 & \\
\hline & $\begin{array}{l}\text { Ambulatory and } \\
\text { bedridden }\end{array}$ & 104(86.0) & $17(14.0)$ & $\begin{array}{l}0.76(0.46- \\
1.26)\end{array}$ & $\begin{array}{l}5.2(1.63- \\
16.67)\end{array}$ & 0.005 \\
\hline \multirow[t]{2}{*}{ Duration on ART } & $\leq 6$ months & 133(88.1) & 18(11.9) & 1.0 & 1.0 & \\
\hline & $6-12$ months & $52(91.2)$ & $5(8.8)$ & $\begin{array}{l}0.70(0.26- \\
1.89)\end{array}$ & $0.83(0.3-2.25)$ & 0.71 \\
\hline
\end{tabular}

all the variables in the descriptive tables are not included here in this table because only variables with $p<0.3$ in the bivariate regression was included. 


\begin{tabular}{|c|c|c|c|c|c|c|}
\hline & 13-24 months & $80(87.0)$ & 12(13.0) & $\begin{array}{l}1.0(0.48- \\
2.07)\end{array}$ & $\begin{array}{l}1.28(0.61- \\
2.70)\end{array}$ & 0.52 \\
\hline & $>24$ months & $495(79.6)$ & $127(20.4)$ & $\begin{array}{l}1.78(1.08- \\
2.91)\end{array}$ & $\begin{array}{l}2.15(1.27- \\
3.63) *\end{array}$ & 0.004 \\
\hline \multirow[t]{2}{*}{$\begin{array}{l}\text { Opportunistic } \\
\text { infection }\end{array}$} & Yes & 182(77.8) & $52(22.2)$ & $1.38(1.0-1.91)$ & $\begin{array}{l}1.68(1.18- \\
2.39) *\end{array}$ & 0.004 \\
\hline & No & 616(83.9) & $118(16.1)$ & 1.0 & 1.0 & \\
\hline \multirow{3}{*}{$\begin{array}{l}\text { Baseline nutritional } \\
\text { status }\end{array}$} & Mild & 186(80.9) & $44(19.1)$ & 1.0 & 1.0 & \\
\hline & Moderate & $543(83.3)$ & 109(16.7) & $\begin{array}{l}0.85(0.60- \\
1.21)\end{array}$ & $\begin{array}{l}0.93(0.65- \\
1.33)\end{array}$ & 0.008 \\
\hline & Severe & $68(80.2)$ & $17(17.8)$ & $\begin{array}{l}1.09(0.62- \\
1.90)\end{array}$ & $\begin{array}{l}1.34(0.62- \\
2.08)\end{array}$ & 0.003 \\
\hline
\end{tabular}

\section{Frequency of relapses of undernutrition}

Data related to the frequency of relapses of undernutrition were only available from September 2012 to July 2016. Data analysis revealed that $170(17.6 \%)$ of adults had relapsed after recovery with $124(72.9 \%)$ relapsing once and 31 (18.3\%) relapsing twice. The remaining, 10 (5.9\%) and 5 (2.9\%) relapsed three and four times respectively after nutritional recovery.

In multivariate logistic regression, statistical significance was declared at $P<0.05$ and place of residence, education, employment status, functional status, presence of anaemia, and baseline nutritional status were statistically significantly associated with frequency of relapse of undernutrition. Individuals who were from rural areas were three times more likely to relapse more than once than those from urban areas. Those who had not attended formal education were more likely to relapse more than once, compared to those who attended secondary education. There was no difference between those attending primary, secondary education and above. Regarding employment, those who were employed were more likely to relapse more than once than those who were unemployed. Individuals who were bedridden were five times more likely to relapse more than once than those who were healthy. Individuals were anaemic at enrolment were nine times more likely to relapse more than once than those who were not. Those who were severely and moderately undernourished at baseline were four and nine times respectively more likely to relapse more than once than those who had mild undernutrition (Table 6). 
Table 6

Determinants of frequency of relapse of undernutrition after nutritional recovery

\begin{tabular}{|c|c|c|c|c|c|c|}
\hline \multirow[t]{2}{*}{ Variables } & & \multirow{2}{*}{ 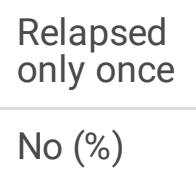 } & Relapsed more & \multirow{2}{*}{$\begin{array}{l}\text { COR }(95 \% \\
\mathrm{Cl})\end{array}$} & \multirow[t]{2}{*}{ AOR(95\%Cl) } & \multirow{2}{*}{$\begin{array}{l}P \text { - } \\
\text { value }\end{array}$} \\
\hline & & & No (\%) & & & \\
\hline \multirow{2}{*}{$\begin{array}{l}\text { Place of } \\
\text { residence }\end{array}$} & Urban & $95(78.5)$ & $26(21.5)$ & 1.0 & 1.0 & \\
\hline & Rural & $29(59.2)$ & $20(40.8)$ & $\begin{array}{l}2.52(1.23- \\
5.16)\end{array}$ & $\begin{array}{l}3.14(1.30- \\
7.57)^{\star}\end{array}$ & 0.011 \\
\hline \multirow[t]{4}{*}{ Marital status } & Single & $17(85.0)$ & $3(15.0)$ & $\begin{array}{l}0.6(0.13- \\
2.72)\end{array}$ & $\begin{array}{l}0.49(0.076- \\
3.19)\end{array}$ & 0.463 \\
\hline & Married & $46(65.7)$ & $24(34.3)$ & $\begin{array}{l}1.74(0.62- \\
4.91)\end{array}$ & $\begin{array}{l}1.50(0.40- \\
5.62)\end{array}$ & 0.551 \\
\hline & Divorced & $41(75.9)$ & $13(24.1)$ & $\begin{array}{l}1.06(0.35- \\
3.19)\end{array}$ & $\begin{array}{l}0.6(0.15- \\
2.35)\end{array}$ & 0.464 \\
\hline & Widowed & $20(76.9)$ & $6(23.1)$ & 1.0 & 1.0 & \\
\hline \multirow[t]{3}{*}{$\begin{array}{l}\text { Educational } \\
\text { status }\end{array}$} & No education & $25(61.0)$ & 16(39.0) & $\begin{array}{l}5.65(1.98- \\
16.19)\end{array}$ & $\begin{array}{l}3.88(1.22- \\
12.36)^{\star}\end{array}$ & 0.022 \\
\hline & Primary & $46(65.7)$ & $24(34.3)$ & $\begin{array}{l}4.61(1.73- \\
12.25)\end{array}$ & $\begin{array}{l}2.23(0.75- \\
6.58)\end{array}$ & 0.150 \\
\hline & $\begin{array}{l}\text { Secondary and } \\
\text { above }\end{array}$ & $53(89.8)$ & $6(10.2)$ & 1.0 & 1.0 & \\
\hline \multirow[t]{2}{*}{$\begin{array}{l}\text { Employment } \\
\text { status }\end{array}$} & Working & $42(53.8)$ & $36(46.2)$ & $\begin{array}{l}7.81(3.44- \\
17.73)\end{array}$ & $\begin{array}{l}3.86(1.15- \\
12.94)^{\star}\end{array}$ & 0.029 \\
\hline & Not working & $82(90.1)$ & $9(9.9)$ & 1.0 & 1.0 & \\
\hline \multirow[t]{2}{*}{$\begin{array}{l}\text { Disclosure of } \\
\text { HIV status }\end{array}$} & Yes & $94(69.6)$ & $41(30.40$ & $\begin{array}{l}2.62(0.95- \\
7.22)\end{array}$ & $\begin{array}{l}2.44(0.79- \\
7.53)\end{array}$ & 0.121 \\
\hline & No & $30(85.7)$ & $5(14.3)$ & 1.0 & 1.0 & \\
\hline \multirow{2}{*}{$\begin{array}{l}\text { Functional } \\
\text { status }\end{array}$} & Working & 117(76.5) & $36(23.0)$ & 1.0 & 1.0 & \\
\hline & $\begin{array}{l}\text { Ambulatory or } \\
\text { bedridden }\end{array}$ & $7(41.2)$ & $10(58.8)$ & $\begin{array}{l}4.64(1.65- \\
13.08)\end{array}$ & $\begin{array}{l}5.21(1.63- \\
16.67)^{\star}\end{array}$ & 0.005 \\
\hline \multirow{2}{*}{$\begin{array}{l}\text { WHO clinical } \\
\text { stage }\end{array}$} & Stage I and II & $89(78.1)$ & $25(21.9)$ & 1.0 & 1.0 & \\
\hline & Stage III and V & $35(62,5)$ & $21(37.5)$ & $\begin{array}{l}2.14(1.06- \\
4.30)\end{array}$ & $\begin{array}{l}1.26(0.45- \\
3.55)\end{array}$ & 0.673 \\
\hline \multirow[t]{2}{*}{ Duration on ART } & $<=6$ months & $9(50.0)$ & $9(50.00$ & $\begin{array}{l}3.38(1.24- \\
9.17)\end{array}$ & $\begin{array}{l}2.51(0.79- \\
8.00)\end{array}$ & 0.121 \\
\hline & $>6$ months & 111(77.1) & $33(22.9)$ & 1.0 & 1.0 & \\
\hline \multirow[t]{2}{*}{$\begin{array}{l}\text { Haemoglobin } \\
\text { level }\end{array}$} & Anaemic & $30(51.7)$ & $28(48.3)$ & $\begin{array}{l}5.54(2.61- \\
11.74)\end{array}$ & $\begin{array}{l}9.28(3.39- \\
25.33) *\end{array}$ & 0.0001 \\
\hline & Not anaemic & $89(85.6)$ & $15(14.4)$ & 1.0 & 1.0 & \\
\hline Cotrimoxazole & Yes & $81(69.2)$ & $36(30.8)$ & $\begin{array}{l}1.91(0.86- \\
4.22)\end{array}$ & $\begin{array}{l}1.86(0.69- \\
5.04))\end{array}$ & 0.223 \\
\hline
\end{tabular}




\begin{tabular}{|c|c|c|c|c|c|c|}
\hline & No & $43(81.1)$ & $10(18.9)$ & 1.0 & 1.0 & \\
\hline \multirow[t]{3}{*}{$\begin{array}{l}\text { Baseline } \\
\text { nutritional status }\end{array}$} & $\begin{array}{l}\text { Mild } \\
\text { malnutrition }\end{array}$ & $39(88.6)$ & $5(11.4)$ & 1.0 & 1.0 & \\
\hline & $\begin{array}{l}\text { Moderate } \\
\text { malnutrition }\end{array}$ & $75(68.8)$ & $34(31.2)$ & $\begin{array}{l}3.54(1.28- \\
9.76)\end{array}$ & $\begin{array}{l}4.30(1.37- \\
13.54)) *\end{array}$ & 0.013 \\
\hline & $\begin{array}{l}\text { Severe } \\
\text { malnutrition }\end{array}$ & $10(58.8)$ & $7(41.2)$ & $\begin{array}{l}5.46(1.43- \\
20.88)\end{array}$ & $\begin{array}{l}9.90(2.04- \\
48.14) *\end{array}$ & 0.004 \\
\hline
\end{tabular}

\section{Results of the qualitative findings}

In the qualitative analysis, food insecurity emerged as one of the key factors that directly and indirectly influenced undernutrition, nutrition program participation and utilisation, and program outcomes - through its impact on weight loss, motivation to enrol in the program, driver of selling and sharing the nutritional support, as a disincentive to graduate from the program and final impact on relapse.

\section{Food insecurity, poor livelihood, poverty and undernutrition}

Adults cited household food insecurity as being a key cause of weight loss. For example;

"Yes, I made an effort to maintain my weight, but your economic status determines your nutritional status. You didn't have enough, then from where can you get? I try [to get] by what I have but my economic status is very low. I know if you eat properly, I will have the energy to work and perform like others but if you are poor, you can do nothing" (Adult female, age 48 \#9).

Poverty and HIV are interwoven, both contributing to food insecurity and undernutrition among people living with HIV. This was particularly reported as an important challenge for people enrolled in the nutritional program. Many adults and caregivers of child participants reported the absence of adequate educational attainment, lack of regular and reliable employment as well as lack of sustainable and reliable income to support them and their family.

"If you are poor and don't have good job, even if you try to create your own job, no one allows you to work for the reason of no education. I asked the kebele administration for job but they told me that you have no education. This makes you very angry. Otherwise, if you have a job, you will not lack adequate and balance food." (Adult female, age 31 \#1).

\section{Food insecurity as a motivation to participate in the nutritional program}

Lack of access to adequate food was one of the key motivations for adults and caregivers to enroll in the nutritional program. Those who lived in urban areas and did not have a reliable source of income or were unemployed reported a lack of access to enough food in their household and that lack of adequate food was a motivation to enroll in the nutritional program. These motivations were shared by both male and female adult participants, reporting circumstances of food shortage in the household. 
If you have something to eat, it should be fine but if you don't have anything to eat like me this food support is very important (Adult male, age 40 \#18).

Yes... many people suffer from lack of access to adequate food. The nutritional support is very helpful to all HIV patients. I have benefited a lot from the nutritional support. So, I suggest this nutritional support to all people with HIV (Adult female, age45 \#2).

\section{Food insecurity as a driver of selling and sharing of the nutritional support}

Household economic problems was one of the main reasons encouraging people to sell and share nutritional support. A health provider and program manager stated that people sell the nutritional support because of their economic conditions and to exchange for other household consumables such as oil or coffee beans:

Instead of eating it [nutritional support] for himself only, they want to sell and exchange it for other household needs such as sugar, salt or oil. Most of the time the reason is this but the base is the poor livelihood condition. So, they are not doing it intentionally, but it is because of their problems (Health provider \#9).

Even though the patients were counseled well, one reason for selling could be the existing economic problems. Poverty by itself would encourage individuals to sell it and spend the money on something that matters to the family is there (Program manager \#1).

Sharing practices were also reported by health providers as common in mothers in circumstances of food insecurity, and caregivers themselves indicated this as highlighted below:

Yes, there is sharing among household members. As far as there is an economic problem, it is not necessarily selling but also sharing. Because if it is given to him, it is likely that the mother will share it with his siblings. If given two sachets then the mother gives one to her other child and keeps one for the HIV positive child (Health provider \#7).

Even though it is prescribed to the sick child if there is no adequate food to eat in the household the mother may share it to other children. So, the mother shares it to fulfill the dietary needs of the other children in the household because she has nothing to give to the other child (Caregiver, age 35 \#9).

"Even now, I can't get enough [food] and I am not taking adequate food. I give everything [including RTUF] I have to my children and my main effort is to feed and care for them. With all the problems I have, I can't get enough food to support myself and my children" (Adult female, age 29 \#15).

\section{Food insecurity, dependence and disincentive to graduate from the program}

Some health providers expressed concerns of dependence of adults and caregivers on the nutritional program due to poverty and food insecurity.

Some patients become very reliant on the supports (soaps, water treatment jerrycan and the nutritional support) given from the health facility because they believe they should get supported due to their HIV condition (Health 
provider \#2)

There were also concerns voiced by health providers that there was reluctance to graduate among some participants enrolled in the nutritional program due to poor socioeconomic status. For this reason, participants preferred prolonged enrolment in the nutritional program to fulfill poor household socioeconomic status:

It is because of their poor economic status that most don't want to graduate. If he graduates, you will not give him Plumpynut in the next time because they don't have other sources of income. Most want their weight to stay as low as possible (Health provider, age 35 \#7).

\section{Food insecurity as a contributor to relapse}

Despite the high magnitude of food insecurity among those enrolled in the nutritional program, health providers identified that there was no remedial strategy as part of the program to prevent relapses of undernutrition among those graduated.

The major issue that creates a problem in this regard is that when they (people living with HIV) graduate from the nutritional program. There is no local or international NGO which we can link people with after their graduation from the program. There is no more NGOs in our area and I don't know the reason. So, there are no efforts made to prevent relapse of malnutrition after nutritional recovery. Because they should be linked into other incomegenerating activities (Health provider, age 27 \#2).

Things that needs improvement in the nutrition program, now after you treat him for malnutrition and he graduates, there is nobody who helps you prevention of relapse. At least you have to link the patients in order the problem not to come again. So, we have big problem in this regard and we don't have supportive organization to do this (Health provider, age 37 \#6).

Some adults indicated the presence of support for participants of the program in rural areas such as provision and maintenance of land as well as initiatives prioritizing adults living with HIV in income-generating activities, but no such experience was reported in patients living in urban areas.

I heard there are some support for people living in rural area. So they should do similar way in urban areas. Living in the city with HIV is very difficult and creates problem of house rent and others (Adult female, age 48 \#9).

However, the above claim may be a perception as no other participant reported such an experience.

\section{Discussion}

This study highlights that household food insecurity coupled with underlying factors such as poverty and poor livelihood undermines the effectiveness of the nutritional programs in HIV care settings, particularly in relation to relapse of undernutrition. Food insecurity was not directly measured in this study but variables which may indirectly relate with it were significantly associated with relapse in the quantitative findings. In addition, the link between food insecurity and the nutritional program emerged during the qualitative data analysis.

Studies in sub-Saharan Africa [37-39] have indicated that people living with HIV are more likely to be unemployed and less skilled. Consistent with the earlier studies, the current study demonstrated high rates of unemployment including from the quantitative sample, and many participants in the qualitative interviews also reporting no or unreliable sources of income related to lack of employment. The lack of employment seemed to place significant stress on 
household income and contributed to food insecurity, and were reported as key motivators for enrolment in the program in the qualitative study.

While both adults and children in the study regained weight and achieved nutritional recovery after enrolment in the nutritional program, sustaining nutritional wellbeing in the presence of household food insecurity was a challenge. In the current study, $18 \%$ of adults and $7 \%$ of children relapsed after nutritional recovery, and $27 \%$ of adults relapsed more than once. These findings are significant as they are an indication of the contribution of demographic, socioeconomic, clinical and nutritional factors to the relapse of undernutrition after nutritional recovery.

The quantitative analysis found that those with lower levels of education were more likely to relapse and that those who did not attend formal education were more likely to relapse more frequently. These are novel findings as we were unable to find previous studies that have examined the relationship between educational status and relapse of undernutrition. It is well acknowledged that a better education is related with better health more generally [40-42], and greater education could be related to enhanced understanding of the program, including the nutritional counseling, and strategies to prevent relapse. Those who were not employed were more likely to replace. This may relate to poor clinical status making work difficult or could also reflect the impact of lower income on food security. Surprisingly, those who were employed were more likely to relapse more than once compared to those who were not employed.

Another study from Ethiopia and Uganda found that people from rural areas have limited access to basic services such as health, education and other social services than their urban counterparts [43]. In the current study being a rural dweller was associated with being more likely to relapse multiple times than their urban counterparts. This suggests the need to consider contextual factors in supporting positive program outcomes.

Another interesting finding is that adults involved in community HIV support groups were less likely to relapse, community HIV support group was not related with the frequency of relapse of undernutrition. This could be related to community HIV support groups' role in reducing food insecurity among people living with HIV and also through improved access to services, they could offer greater support in maintaining weight and reducing HIV related stigma $[44,45]$.

In addition, clinical and nutritional factors such as duration on ART, presence of opportunistic infections and anaemia were associated with relapse of undernutrition. For example, anaemia among people living with HIV is common due to the HIV illness and some ART medications $[46,47]$ and in the current study, those with anaemia and severe and moderate acute undernutrition at enrolment were more likely to relapse. Those with the worst clinical conditions (bedridden functional status) were also more likely to relapse more than once than those apparently healthy. These clinical and nutritional conditions may contribute to undernutrition directly because worst HIV infection contributes to undernutrition [48], and also indirectly by limiting the individual from contributing economically leading to poverty and food insecurity. This is consistent with literature which states that prolonged illnesses are one of the essential contributors of poverty and food insecurity among people living with HIV [11, 49]. However, there are no other studies demonstrating the role of these clinical and nutritional characteristics in contributing to relapse of undernutrition after nutritional recovery among those enrolled in the nutritional programs.

While undernutrition in people living with HIV is underpinned by underlying determinants of food insecurity such as poverty, poor livelihood, it seems that the nutritional program primarily focuses on short term treatment of undernutrition, dealing with "tip of the iceberg". As a result, the effectiveness of the nutritional program in improving nutritional status is hampered, including the reluctance of people to graduate from the program. 
Similarly, sharing and selling of the nutritional support was a common practice and appeared to be driven by food insecurity and its underlying determinants. In the current study, adults and caregivers shared the nutritional support to fulfill dietary needs of their family due to lack of adequate food in the households. These findings support a study conducted in Ethiopia which reported RUTF sharing was more common in poor households indicating the negative impact of food insecurity on the nutritional program [29]. Although no program participants reported selling the nutritional support, health providers stated that this happened and did so for families to fulfill family nutritional needs. Selling and sharing undermine the effectiveness of the program through reducing and changing ration size.

While the study has provided important qualitative and quantitative evidence regarding the predictors of relapse and the role of food insecurity, there were a number of limitations. Food insecurity was not measured directly in the quantitative data. Hence, the relationship between food insecurity and relapse of undernutrition may not be a direct and quantifiable relationship. The other limitations include that it was not possible to validate the accuracy and precision of measurement of the demographic, socioeconomic, clinical and nutritional characteristics as only secondary data was used. It is also likely that the originality of information in the qualitative study may have been lost during the translation and transcription, even though efforts were made to maintain the original conversation as narrated by the participants.

\section{Conclusion And Recommendation}

In conclusion, a significant proportion of adults and children relapsed after nutritional recovery and qualitative findings highlighted that food insecurity, poverty, and poor livelihood were common among people living with HIV enrolled in the nutritional program. Food insecurity contributed to the problems of effectiveness of the nutritional program in three ways including:(i) many adults and caregivers were motivated to join the nutritional program because of the food insecurity, (ii) Individuals from food-insecure households shared and sold the nutritional support to fulfill family dietary needs, and (iii) a significant number of adults and children relapsed because after graduation they were food insecure, leading to the loss of weight that was gained from the program. Various demographic characteristics such as low educational status, employment status, rural residence and involvement in a community support group; clinical characteristics such as poor clinical condition and presence of opportunistic infections; and nutritional characteristics such as moderate and severe acute undernutrition all were associated with relapse of undernutrition. The above factors likely contributed to a vicious cycle of complex poor nutritional outcomes. Thus, addressing poverty and poor livelihood is crucial to improve and sustain the nutritional wellbeing of people living with HIV enrolled in nutritional programs. Nutritional programs in HIV care should be poverty oriented and should consider household food security status in their program implementation. To improve the overall nutritional wellbeing and HIV treatment outcomes in these programs, they need to address the underlying social and economic determinants of undernutrition.

\section{List Of Abbreviations}

ART: Antiretroviral Therapy

BMI: Body Mass Index

CD4: Cluster Differential T-lymphocytes

Cl: Confidence Interval

ERC Ethical review committee

HIV/AIDS: Human Immune Virus / Acquired Immune Deficiency

Page 21/25 
MAM: Moderate Acute Undernutrition

MUAC: Mid Upper Arm Circumference

P: P-value

RUSF: Ready to Use Supplementary Food

RUTF: Ready to Use Therapeutic Food

SAM: Severe Acute Malnutrition

SBRE: Social and Behavioural Research Ethics

TB: Tuberculosis

W/H\%: Weigh for Height z-scores

WHO: World health organization

WHZ: Weight for Height z-scores

\section{Declarations}

Ethical issues: There were no direct ethical concerns regarding the quantitative data, but issues of anonymity and confidentiality were sources of concern in the qualitative study. To address these concerns for the qualitative study participants, strategies such as reassurance, maintenance of confidentiality and anonymity was employed. In addition, qualitative study participants were provided with compensation for the time they spent during the interview. Ethical clearance was also secured from the Flinders University Social and Behavioural Research Ethics Committee (SBREC) (7118) in Australia and Mekelle University Ethical review committee (ERC 06211/2016), Ethiopia.

Consent to participant: Written informed consent was obtained from all study participants.

Consent for publication: Not applicable.

Availability of data and material: The datasets used and/or analysed during the current study are not possible to share with a third party because of the agreement to only use it for the current study. However, the authors can share deidentified aggregate data.

Competing interests: The authors declare that they have no competing interests.

Funding: This study was part of a PhD thesis and the Flinders University of South Australia covered the tuition fee, data collection, and overseas travel expenses during the PhD candidature. Flinders University had no involvement in the design, collection, analysis, interpretation, writing and the publication of this manuscript.

Authors' contributions: FT designed the study, collected, analysed and interpreted the data and drafted the manuscript. AZ, SJ, and LM critically reviewed the design, analysis and interpretation of data and contributed to the manuscript. 
This manuscript is taken from $\mathrm{PhD}$ thesis of the first author. We would like to acknolwedge the Australian Government Research Training program and Flinders University for covering the tuition fee, living expenses and fieldwork and overseas travel costs during the PhD candidature. We would also like to express our gratitude to Mekelle University, Tigray Regional Health Bureau and the study hospitals (Mekelle, Shul and Lemlem Karl) for providing administrative support and facilitating the data collection process, and also the quantitative data collectors in these hospitals. The qualitative study participants also deserve special thanks for sharing their personal studies.

\section{References}

1. Pinstrup-Andersen P: Food security: definition and measurement. Food security 2009, 1(1):5-7.

2. Smith LC, Alderman H, Aduayom D: Food insecurity in sub-Saharan Africa: new estimates from household expenditure surveys, vol. 146: Intl Food Policy Res Inst; 2006.

3. Swindale A, Bilinsky P: Development of a Universally Applicable Household Food Insecurity Measurement Tool: Process, Current Status, and Outstanding Issues. The Journal of Nutrition 2006, 136(5):1449S-1452S.

4. Bukusuba J, Kikafunda JK, Whitehead RG: Food security status in households of people living with HIV/AIDS (PLWHA) in a Ugandan urban setting. British Journal of Nutrition 2007, 98(1):211-217.

5. Weiser SD, Tuller DM, Frongillo EA, Senkungu J, Mukiibi N, Bangsberg DR: Food insecurity as a barrier to sustained antiretroviral therapy adherence in Uganda. PloS one 2010, 5(4):e10340.

6. Nagata JM, Magerenge RO, Young SL, Oguta JO, Weiser SD, Cohen CR: Social determinants, lived experiences, and consequences of household food insecurity among persons living with HIV/AIDS on the shore of Lake Victoria, Kenya. AIDS care 2012, 24(6):728-736.

7. Tiyou A, Belachew T, Alemseged F, Biadgilign S: Food insecurity and associated factors among HIV-infected individuals receiving highly active antiretroviral therapy in Jimma zone Southwest Ethiopia. Nutrition journa/2012, 11(1):51.

8. Weiser SD, Fernandes KA, Brandson EK, Lima VD, Anema A, Bangsberg DR, Montaner JS, Hogg RS: The association between food insecurity and mortality among HIV-infected individuals on HAART. Journal of acquired immune deficiency syndromes (1999) 2009, 52(3):342.

9. Musumari PM, Wouters E, Kayembe PK, Nzita MK, Mbikayi SM, Suguimoto SP, Techasrivichien T, Lukhele BW, ElSaaidi C, Piot P: Food insecurity is associated with increased risk of non-adherence to antiretroviral therapy among HIV-infected adults in the Democratic Republic of Congo: a cross-sectional study. PloS one 2014, 9(1):e85327.

10. Singer AW, Weiser SD, McCoy SI: Does food insecurity undermine adherence to Antiretroviral Therapy? a systematic review. AIDS and Behavior 2015, 19(8):1510-1526.

11. Weiser SD, Young SL, Cohen CR, Kushel MB, Tsai AC, Tien PC, Hatcher AM, Frongillo EA, Bangsberg DR: Conceptual framework for understanding the bidirectional links between food insecurity and HIV/AIDS-. The American journal of clinical nutrition 2011, 94(6):1729S-1739S.

12. Gillespie S: Poverty, Food Insecurity, HIV Vulnerability and the Impacts of AIDS in sub? Saharan Africa. 2008.

13. Fauk NK, Mwakinyali SE, Putra S, Mwanri LJIJoHRiH: The socio-economic impacts of AIDS on families caring for AIDS-orphaned children in Mbeya rural district, Tanzania. 2017, 10(2):132-145.

14. Himmelgreen DA, Romero-Daza N, Turkon D, Watson S, Okello-Uma I, Sellen D: Addressing the HIV/AIDS-food insecurity syndemic in sub-Saharan Africa. African Journal of AIDS Research 2009, 8(4):401-412.

15. Castleman T, Diene S: The Role of NACS in Health Systems. 2015. 
16. Scarcella P, Buonomo E, Zimba I, Doro Altan A, Germano P, Palombi L, Marazzi M: The impact of integrating food supplementation, nutritional education and HAART (Highly Active Antiretroviral Therapy) on the nutritional status of patients living with HIV/AIDS in Mozambique: result from the DREAM programme. Igiene e sanita pubblica 2011, 67(1):41-53.

17. Sackey JD: Implementation and impact of a nutrition assessment, counseling and support program on people living with HIV in Accra, Ghana. Tufts University, Gerald J. and Dorothy R. Friedman School of Nutrition Science and Policy; 2016.

18. Rawat R, Faust E, Maluccio JA, Kadiyala S: The impact of a food assistance program on nutritional status, disease progression, and food security among people living with HIV in Uganda. JAIDS Journal of Acquired Immune Deficiency Syndromes 2014, 66(1):e15-e22.

19. Young S, Wheeler AC, McCoy SI, Weiser SD: A review of the role of food insecurity in adherence to care and treatment among adult and pediatric populations living with HIV and AIDS. AIDS and Behavior 2014, 18(5):505515.

20. Cantrell RA, Sinkala M, Megazinni K, Lawson-Marriott S, Washington S, Chi BH, Tambatamba-Chapula B, Levy J, Stringer EM, Mulenga L: A pilot study of food supplementation to improve adherence to antiretroviral therapy among food insecure adults in Lusaka, Zambia. Journal of acquired immune deficiency syndromes (1999) 2008, 49(2).

21. Tirivayi N, Koethe JR, Groot W: Clinic-based food assistance is associated with increased medication adherence among HIV-infected adults on long-term antiretroviral therapy in Zambia. Journal of AIDS \& clinical research 2012, 3(7):171.

22. Asnakew MJOALJ: Food Insecurity: Prevalence and Associated Factors among Adult Individuals Receiving Highly Active Antiretroviral Therapy (HAART) in ART Clinics of Hosanna Town, Hadiya Zone, Southern Ethiopia. 2015, 2(1800):1-9.

23. Sholeye OO, Animasahun VJ, Salako AA, Oyewole BK: Household food insecurity among people living with HIV in Sagamu, Nigeria: A preliminary study. Nutrition and Health 2017, 23(2):95-102.

24. Tsai AC, Bangsberg DR, Emenyonu N, Senkungu JK, Martin JN, Weiser SD: The social context of food insecurity among persons living with HIV/AIDS in rural Uganda. Social Science \& Medicine 2011, 73(12):1717-1724.

25. Sadler K, E. Bontrager, Rogers B, J. Coates SG, Y. Kidane: Food by Prescription: Measuring the impact and costeffectiveness of prescribed food on recovery from malnutrition and HIV disease progression among HIV+ adult clients in Ethiopia. In.: Feinstein International Center, Friedman School of Nutrition Science and Policy, Tufts University, Boston, USA; 2012.

26. Nagata JM, Cohen CR, Young SL, Wamuyu C, Armes MN, Otieno BO, Leslie HH, Dandu M, Stewart CC, Bukusi EA et al: Descriptive characteristics and health outcomes of the food by prescription nutrition supplementation program for adults living with HIV in Nyanza Province, Kenya. Plos One 2014, 9(3).

27. Dibari F, Bahwere P, Le Gall I, Guerrero S, Mwaniki D, Seal A: A qualitative investigation of adherence to nutritional therapy in malnourished adult AIDS patients in Kenya. Public Health Nutr 2012, 15(2):316-323.

28. Olsen M, Markos T, Pernille K, Henrik F, Lotte H: Use, perceptions, and acceptability of a ready-to-use supplementary food among adult HIV patients initiating antiretroviral treatment: a qualitative study in Ethiopia. Patient Preference and Adherence 2013, 2013(7).

29. Hussien S, Worku A, Aklilu A, Abate K: Ready-to-Use Therapeutic Food for management of wasting in HIV infected adults: qualitative investigation of views and experiences of patients in Ethiopia. International Journal of Nutrition and Food Sciences 2015; 2015, 4(5):518-529. 
30. Olsen M, Markos T, Pernille K, Henrik F, Lotte H: Use, perceptions, and acceptability of a ready-to-use supplementary food among adult HIV patients initiating antiretroviral treatment: a qualitative study in Ethiopia. Patient Preference and Adherence 2013, 2013(7).

31. Tigray Regional Health Bureau: Tigray Regional Health Bureau 2007 Ethiopian fisical year annual Profile. In. Mekelle, Ethiopia; 2015.

32. Ethiopian Federal Ministry of Health: Ethiopian Guide to Clinical Nutrition Care for Children and Adults Living with HIV. In.; 2008.

33. Small ML: How to conduct a mixed methods study: Recent trends in a rapidly growing literature. Annual review of sociology 2011, 37.

34. Creswell JW, Plano Clark VL, Gutmann ML, Hanson WE: Advanced mixed methods research designs. Handbook of mixed methods in social and behavioral research 2003, 209:240.

35. Tashakkori A, Creswell JW: The new era of mixed methods. In.: Sage Publications; 2007.

36. Gale NK, Heath G, Cameron E, Rashid S, Redwood S: Using the framework method for the analysis of qualitative data in multi-disciplinary health research. BMC Medical Research Methodology 2013, 13:117-117.

37. Sprague L, Simon S, Sprague CJAJoAR: Employment discrimination and HIV stigma: survey results from civil society organisations and people living with HIV in Africa. 2011, 10(sup1):311-324.

38. Grossman Cl, Stangl AL: Global action to reduce HIV stigma and discrimination. 2013, 16(3S2):18881.

39. Sprague L, Simon S, Sprague C: Employment discrimination and HIV stigma: survey results from civil society organisations and people living with HIV in Africa. African Journal of AIDS Research 2011, 10(sup1):311-324.

40. Conti G, Heckman J, Urzua SJAER: The education-health gradient. 2010, 100(2):234-238.

41. WHO Commission on Social Determinants of Health: Closing the gap in a generation: health equity through action on the social determinants of health: final report of the commission on social determinants of health. 2008.

42. Marmot M, Friel S, Bell R, Houweling TA, Taylor S, Health CoSDo: Closing the gap in a generation: health equity through action on the social determinants of health. The lancet 2008, 372(9650):1661-1669.

43. Cramer C, Oya C: Identifying the most deprived in rural Ethiopia and Uganda: a simple measure of socio-economic deprivation AU - Sender, John. Journal of Eastern African Studies 2018, 12(3):594-612.

44. Kabore I, Bloem J, Etheredge G, Obiero W, Wanless S, Doykos P, Ntsekhe P, Mtshali N, Afrikaner E, Sayed R: The effect of community-based support services on clinical efficacy and health-related quality of life in HIV/AIDS patients in resource-limited settings in sub-Saharan Africa. AIDS patient care and STDs 2010, 24(9):581-594.

45. Kaschula S: Using People to Cope with the Hunger: Social Networks and Food Transfers Amongst HIV/AIDS Afflicted Households in KwaZulu-Natal, South Africa. AIDS and Behavior 2011, 15(7):1490.

46. Gedefaw L, Yemane T, Sahlemariam Z, Yilma D: Anemia and risk factors in HAART naïve and HAART experienced HIV positive persons in South West Ethiopia: a comparative study. PLOS ONE 2013, 8(8):e72202.

47. Jaganath D, Walker AS, Ssali F, Musiime V, Kiweewa F, Kityo C, Salata R, Mugyenyi P, DART, Trials A: HIVassociated anemia after 96 weeks on therapy: determinants across age ranges in Uganda and Zimbabwe. AIDS research and human retroviruses 2014, 30(6):523-530.

48. WHO: WHO case definitions of HIV for surveillance and revised clinical staging and immunological classification of HIV-related disease in adults and children. 2007.

49. Anema A, Vogenthaler N, Frongillo EA, Kadiyala S, Weiser SD: Food insecurity and HIV/AIDS: Current knowledge, gaps, and research priorities. Current HIV/AIDS Reports 2009, 6(4):224-231. 